\title{
Modeling Dynamics in the Central Regions of Disk Galaxies
}

\author{
Isaac Shlosman \\ Department of Physics \& Astronomy, University of Kentucky, Lexington, KY 40506-0055, USA
}

\begin{abstract}
The central regions of disk galaxies are hosts to supermassive black holes whose masses show a tight correlation with the properties of surrounding stellar bulges. While the exact origin of this dependency is not clear, it can be related to the very basic properties of dark matter halos and the associated gas and stellar dynamics in the central kpc of host galaxies. In this review we discuss some of the recent developments in modeling the wide spectrum of dynamical processes which can be affiliated with the above phenomena, such as the structure of molecular tori in AGN, structure formation in triaxial halos, and dissipative and non-dissipative dynamics in nested bar systems, with a particular emphasis on decoupling of gaseous nuclear bars. We also briefly touch on the subject of fueling the nuclear starbursts and AGN.
\end{abstract}

\section{INTRODUCTION}

There is a natural tendency to extrapolate known conditions and evolution beyond their original limits. A somewhat similar situation has happened in galactic dynamics when processes operating on large spatial scales have been postulated to play a similar role within the central kpc with only a minor correction for shorter timescales. However, during the last decade it became apparent that a number of fundamental differences exist between galactic disks at large and their central regions. These differences provide an ample evidence that the traditional roles of various dynamic agents can change in the very galactic interiors. The corollary is that a large dynamic range, even when limited to within the central kpc only, results in a plethora of phenomena so diverse in their nature that various new techniques are required to address the overall evolution in the region. In other words, the central kpc of disk galaxies is an entangled web of complex processes whose understanding will depend on high resolution observations and state-of-the-art numerical modeling, and no single observational or modeling technique can provide an adequate description of the processes thought. Here we review some of processes thought to dominate the region from a fraction of a pc to a $\mathrm{kpc}-$ about 4 orders of magnitude in spatial scale, and describe the present status of their modeling. What are the most important factors within this region that can influence its evolution?

First, the central kpc of a fully-grown galactic disk appears to be dominated by the baryonic matter (e.g., McGaugh, Rubin \& de Block 2001; de Block \& Bosma 2002; Bosma 2002), while beyond the $\sim 10 \mathrm{kpc}$ scale the dark matter (DM) prevails. Universally obtained numerical cuspy density profiles in CDM halos (e.g., Navarro, Frenk \& White 1996, hereafter NFW) could be flattened during a stage of inhomogeneous baryonic inflow toward the central regions (El-Zant, Shlosman \& Hoffman 2001). It is 
possible that the size of the flat core in the DM halo has evolved in conjunction with the growing galactic disk and is heavily influenced by the merger history. How much dissipation has been involved in the formation of inner disks and bulges has yet to be determined, but it seems highly unlikely that their origin was purely collisionless.

Second, the central supermassive black holes (SBHs) situated in galactic centers serve as another defining characteristic of these regions. The SBHs appear ubiquitous and the observed tight correlation between their masses and the dispersion velocities in the galactic bulges has verified unambiguously that host galaxies are aware of their central constituents on the smallest spatial scales, and vice versa (e.g., review by Ferrarese \& Ford 2004). This correlation is strikingly at odds with the small radius of influence of the $\mathrm{SBH}, r_{\mathrm{SBH}} \sim 5 M_{\bullet}, 7 \sigma_{100}^{-2} \mathrm{pc}$, where $M_{\bullet, 7}$ is the SBH mass in units of $10^{7} \mathrm{M}_{\odot}$ and $\sigma_{100}$ is the velocity dispersion in the bulge in $100 \mathrm{~km} \mathrm{~s}^{-1}$. Note, that the SBH interaction with the central kpc of galaxies is twofold: radiative (when it is in the active galactic nucleus $[\mathrm{AGN}]$ phase) and gravitational (section 3).

Third, large-scale galactic disks often harbor rapidly rotating stellar bars — those which extend to their corotation radii (e.g., Athanassoula 1992). The corresponding properties of nuclear bars are much less known, but it is clear that they can be either star or gas dominated (e.g., review by Shlosman 2001), and are not necessarily fast rotators (section 4). Because bars frequently have a decisive effect on disk dynamics and evolution, we shall pay special attention to bar-induced dynamics in this review.

Next, the surface density and the amounts of cold (molecular) gas generally increase towards the galactic center, and this trend is more profound in barred galaxies (Sakamoto et al. 1999; Jogee, Scoville \& Kenney 2004). Moreover, there are clear indications that the state of molecular gas in the center differs from that in the outer disk in such a way that the fraction of diffuse (unbound) molecular gas is much higher, and its dense part is both denser and warmer (e.g., Hüttemeister 2002). The gas at the very center of AGN host galaxies, the inner few pc from the SBHs, is detected in the form of molecular tori (e.g., Antonucci 2002) and a number of alternative explanations to the existence of these geometrically thick configurations are known. We discuss these issues in section 2 , and only note here that the vertical structure of these tori can be heavily influenced by magnetic fields amplified in the underlying sheared accretion flow and modulated by the AGN radiation field.

The structure of this paper is as follows. In the next section we discuss the gas dynamics on the smallest relevant scales, $\sim$ pc. Section 3 analyzes the SBH-bulge relation within the context of triaxial background potentials. New developments in nested bar dynamics are addressed in section 4, and some aspects of AGN fueling are given in section 5. Finally, section 6 deals with the possible causal connection between the nuclear starbursts and AGN.

\section{MODELING MOLECULAR TORI IN AGN: DISK STARBURSTS $V S$ DISK WINDS}

The central engine in AGN is surrounded by a dusty torus and is visible only in the poleon viewing of "type 1" sources and blocked from view in edge-on "type 2" sources. 
Compelling evidence for the toroidal orientation-dependent obscuration comes from both the optical, especially line spectropolarimetry (e.g., Antonucci 2002), and X-ray regimes. The torus reveals itself also in dust IR emission, including the recent direct $K$-band imaging of the inner region in NGC 1068 (Weigelt et al. 2004) and is likely to consist of a large number of individually very optically thick dusty clouds (Krolik \& Begelman 1988). A serious impediment to detailed dynamical calculations of the torus structure has been the lack of guidance from realistic radiative transfer models based on IR observations. A formalism to handle dust clumpiness has been developed only recently, and shows that it resolves fundamental difficulties encountered by previous theoretical efforts (Nenkova, Ivezic \& Elitzur 2002; Elitzur, Nenkova \& Ivezic 2004). Clumpy models have since been employed in a number of observational studies, including that of Spitzer observations by the GOODS Legacy project (Treister et al. 2004).

Beyond the original weakly self-gravitating cloud model of Krolik \& Begelman (1988), two alternative scenarios have been promoted in order the explain the geometrically-thick molecular tori in AGN — the hydrostatic model where the vertical 'puffing' is performed by supernova ( $\mathrm{SN}$ ) injected energies (Wada \& Norman 2002, Wada \& Tomisaka 2005), and the accretion disk wind model with a combined MHD/radiative driving force (Emmering, Blandford \& Shlosman 1992; Königl \& Kartje 1994; Bottorff et al. 1997; 2000; Kartje, Königl \& Elitzur 1999).

Numerical simulations which have been modeling such SN explosions as an energy source (Wada \& Norman 2002) require an oversized torus of $\sim 100$ pc, which exceeds the upper limits on the mid-IR sizes of $\sim 30 \mathrm{pc}$ in both NGC 1068 (Bock et al. 2000) and NGC 4151 (Radomski et al. 2003). In such a model, much of the IR emission will come from outer boundary, while observations favor the inner boundary dominated tori. The starbursts in the composite Seyfert 2 nuclei discussed in section 6 are even more extended and clearly are not associated with the tori. In the hydrostatic model, the bulk of the energy in the torus is found in the cloud motions and the problem lies in the efficient cooling by cloud collisions rather than in insufficient viscous heating.

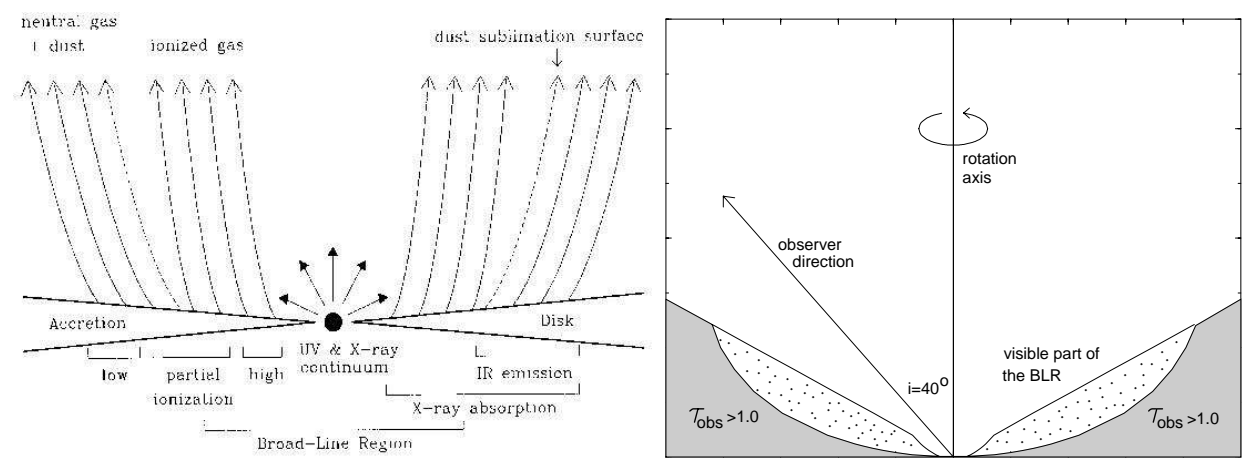

FIGURE 1. Molecular tori from disk hydromagnetic wind in AGN (obscuring torus is the gray-shaded region where $\tau_{\mathrm{obs}}>1$ ). Left: from Kartje \& Königl (1994). Right: specific application to NGC 5548 (from Bottorff et al. 1997).

On the other hand, winds fit naturally within the theoretical framework for AGN (Fig. 1). Bipolar structures are associated with accretion disks on all scales, from young stellar objects to quasars, and it is natural to assume that they are symbiotically related to the dynamical outflows from these objects. Most important, it is entirely possibly that 
these outflows provide a natural channel for the angular momentum loss by the accreting material and hence can be naturally affiliated with magnetic fields (Blandford \& Payne 1982; Emmering et al. 1992). Such outflows from accretion disks in AGN can possess substantial optical depths, depending on their ability to uplift the cold material, and are clumpy. The optically thick part of the wind meets all the requirements of the AGN torus, suggesting itself as a natural alternative to the problematic hydrostatic paradigm. Note that strong radiation fields generated in the vicinity of the SBH are inevitably dynamically important, and accretion disks are prime sources of substantial magnetic fields as well (Arav, Shlosman \& Weymann 1997 and refs. therein). It seems increasingly plausible that magnetic fields which provide the source of viscosity within the AGN disks (e.g., Hawley, Gammie \& Balbus 1996; Hawley, Balbus \& Stone 2001), can also serve as a channel for angular momentum loss by these disks to the ambient medium (Blandford \& Payne 1982; Emmering et al. 1992; Contopoulos 1995). Moreover, the amount of radiation momentum "missing" in the absorption troughs of BAL QSOs is of the same order as the estimated flow momentum, suggesting that radiation pressure probably also plays a key role in accelerating the outflow. Lastly, at least in the BAL QSOs the absorbing column in X-rays and UV consists of intrinsically clumpy material (e.g., Mathur, Elvis \& Singh 1995; Shields \& Hamann 1997) and its column density is comparable with that of the torus (Mathur et al. 2000). Elitzur \& Shlosman (in preparation) have combined the wind kinematics with the IR radiative transfer, thus constraining the parameter space of possible solutions and providing a critical comparison with the latest observations — the characteristic size of the torus, $\sim 10 \mathrm{pc}$, appears to be consistent with mid-IR observations of tori in nearby Sy 2 galaxies.

\section{MODELING $M_{\bullet}-\sigma$ : DYNAMICS IN TRIAXIAL POTENTIALS}

This correlation provides the strongest evidence so far that the SBHs in galactic nuclei are fueled via global sources rather than the local ones. However, the details of this process are obscure: one can roughly divide the possible explanations of this 'conspiracy' into gasdynamical or stellar dynamical, and it is entirely possible that the fully selfconsistent model should incorporate both frameworks. However, here we put the emphasis on the stellar dynamics because it allows to relate the properties of the DM halos with those at the smallest spatial scales in the vicinity of the SBHs where most probably the gas dynamics in combination with radiation pressure plays a more dominant role.

Halos identified in cosmological simulations are invariably found to be triaxial (e.g., Warren et al. 1992; Cole \& Lacey 1996) with density median axial ratios of 0.5 - 0.6. Their asymmetries are expected to be partially 'washed-out,' since it has been shown (e.g., Dubinski 1994) that the settling of a baryonic component can significantly reduce the initial nonaxisymmetry born of dissipationless collapse. From an observational standpoint, radial profiles of the DM halos in fully formed galaxies tend to have nearly constant density cores (e.g., Flores \& Primack 1994; Burkert 1995; Kravtsov et al. 1998; Boriello \& Salucci 2001; de Block \& Bosma 2002). A similar effect has recently been inferred for clusters of galaxies (Sand et al. 2002). Theoretically, dissipationless

CDM simulations of galactic halos agree with a universal density profile $\propto r^{-\beta}$, where 
$\beta=1-1.5$ (e.g., Dubinski \& Carlberg 1991; Warren et al. 1992; Cole \& Lacey 1996; Fukushige \& Makino 1997; Moore et al. 1999; Klypin et al. 2000). NFW have found a fitting formula for the density profile of DM halos, for a wide range of cosmological models, in which the inner profile diverges as $r^{-1}$, while the outer profile drops as $r^{-3}$. A cuspy density profile arises inherently from the cold gravitational collapse in an expanding universe (Lokas \& Hoffman 2000). The CDM model, therefore, predicts that the inner density profile of galactic scale DM halos is characterized by a density cusp while observations of the dynamics of the central regions of galaxies imply a core-halo structure of the DM. This controversy between observations of DM cores and density cusps in numerical models is not a fundamental one and can be resolved within the general context of CDM cosmology (El-Zant et al. 2001).

It is interesting that the orbital structure of the inner regions of slowly rotating, asymmetric potentials with flat (i.e., harmonic) cores is dominated by box orbits (e.g., Lake \& Norman 1983; Binney \& Tremaine 1987), which have no particular sense of circulation. They are self-intersecting and cannot be populated with gas. Dissipation causes material to sink quickly toward the center. In the process, interaction with the triaxial harmonic core causes the baryonic material to lose most of its angular momentum. One can conjecture, that as the collapsing gas becomes self-gravitating, i.e., when its density exceeds that of a background DM, runaway star formation will follow, leading to the possible formation of a (classical) bulge. El-Zant et al. (2003) have noticed that this also happens to be the criterion for the destruction of the harmonic core and the emergence of loop (or tube) orbits, which do have a definite sense of circulation. The role of the $\mathrm{SBH}$ is to contribute to the emergence of these orbits in the very central region. It is the collusion between the SBH and the more extended hot baryonic component in creating the loop orbits that leads to the observed correlations, $M_{\bullet}-M_{\mathrm{b}}$ and $M_{\bullet}-\sigma_{\mathrm{b}}$.

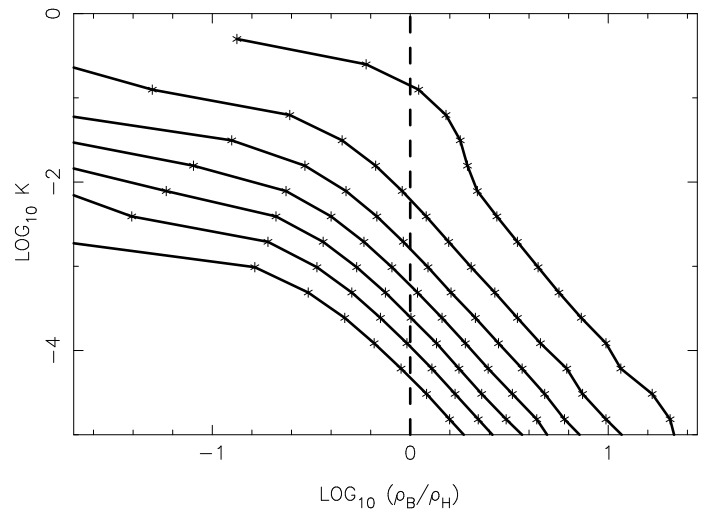

FIGURE 2. SBH-to-bulge mass ratio $K$ for different $p_{\text {crit }}$ (see text for definition) as a function of the bulge-to-halo core density ratio required to create closed loop orbits with axis ratios $p \geq p_{\text {crit }}$ within the bulge core, $R<R_{\mathrm{b}}$. The mass of the bulge core, $M_{\mathrm{b}}$ is chosen to satisfy $p>p_{\text {crit }}$ everywhere outside the bulge. From left to right, the characteristic $p_{\text {crit }}$ associated with a given curve increases from 0.3 to 0.9 . The bulge core density $\rho_{\mathrm{b}}$ is varied by fixing $M_{\mathrm{b}}$ and changing $R_{\mathrm{b}}$. The vertical dashed line at $\rho_{\mathrm{b}} / \rho_{\mathrm{H}}=1$ is the approximate boundary between the self- and non-self-gravitating regimes in the bulge. For densities $\rho_{\mathrm{b}} / \rho_{\mathrm{H}} \sim 1$, the $p_{\text {crit }}=0.9$ curve corresponds to an (unrealistically) overmassive $\mathrm{SBH}$ and is shown for comparison only. The range of $K$ values, for these densities, thus is limited to within $10^{-4}-10^{-2}$ (El-Zant et al. 2003). 
Fig. 2 summarizes the SBH-to-bulge mass ratios, $K$, predicted by the model as a function of a bulge-to-halo core density ratio, $\rho_{\mathrm{b}} / \rho_{\mathrm{H}}$ for the halo potential axial ratio of 0.9 . The seven curves shown differ in the value of $p_{\text {crit }}$ - the critical value of axial ratios of loop orbits above which the gas circulation can be sustained for secular timescales. The value of $p_{\text {crit }}$ appears to be in the range of $\sim 0.4-0.5$, based on numerical simulations of Smoothed Particle Hydrodynamics (SPH) in a fixed background potential, for both non- and self-gravitating gas, with the isothermal equation of state (Pichardo \& Shlosman 2005). This means that the model predicts a SBH-to-bulge mass ratio within the observed range of $K \sim 10^{-4}-10^{-2}$, based on the mix of gas- and stellar dynamical processes within triaxial DM halos.

While this approach is promising, the real challenge is to incorporate it within the general framework of galaxy formation and evolution. Processes like galaxy mergers and dissipative dynamics affect both the DM halo shapes and their radial density profiles, as well as the growth of the SBHs. It is the tightness of the $M_{\bullet}-\sigma_{\mathrm{b}}$ correlation across a vast dynamic range which is puzzling.

\section{MODELING NESTED BARS: DISSIPATIVE AND NON-DISSIPATIVE DYNAMICS}

The large fraction of stellar bars in the local universe has been noticed already by Hubble (1936; e.g., tuning fork), and their importance for understanding dynamics in disk galaxies has been widely recognized (e.g., Athanassoula 1984). But the exact role of bars in cosmological evolution is only now beginning to be unraveled. The claim that bars disappear at redshifts above 0.5 (Abraham et al. 1999) has not been substantiated. The GEMS survey (Rix et al. 2004) has just shown that the fraction of strong bars remains unchanged up to redshifts of $\sim 1$ at least, with their size and ellipticity distributions being compatible with a mild evolution not driven by major mergers (Jogee et al. 2004b). The main point of interest here is that these stellar bars have an effect on the mass redistribution in a galaxy, specifically, they induce gas inflows to the central kpc, which is observed (e.g., Jogee 2004) and modeled numerically, both neglecting the gas self-gravity (e.g., Combes \& Gerin 1985) and accounting for it (e.g., Simkin, Sue \& Schwarz 1980; Schwarz 1984; Athanassoula 1992; Shlosman \& Noguchi 1993; Friedli \& Martinet 1993; Heller \& Shlosman 1994). Typically, the most direct dynamical effect bars have on the host galaxy is by means of gravitational torques and resonances. Unfortunately, the bar torques have little effect within the central kpc where the underlying gravitational potential becomes nearly axisymmetric. The outer inner Lindblad resonance (OILR), if this exists, usually borders this region and the observed nuclear spirals (e.g., Laine et al. 1999) which can be driven by the bar are too weak to trigger any inflow (Englmaier \& Shlosman 2000).

This is why the existence of a different species - nuclear bars, confined within the inner kpc region, is so important for the evolution of galactic centers. In this section we review the few possibilities which lead to a nonaxisymmetric mass distribution in these regions and focus on the gas inflow. 


\subsection{Stellar and gaseous secondary bars: observations}

Nuclear bars almost always come in conjunction with large-scale bars (Laine et al. 2002) - a clear indication that this is a prerequisite for their existence, although one can envisage a scenario where they form separately. We, therefore, follow the established prescription and call the large-scale bars 'primary,' and the nuclear bars - secondary. The secondary bars can be pure stellar, gaseous or of mixed content. Evolutionary patterns will differ in all three cases.

Systems of two nested bars which involve a stellar secondary bar appear to be unexpectedly abound in the local universe - about $1 / 3$ of all barred disks are hosts to these configurations (Laine et al. 2002; Erwin \& Sparke 2002). Comparable statistics of gaseous or gas-dominated secondary bars is unavailable currently. The detection of stellar nuclear bars is limited to optical and NIR photometry so far. Laine et al. (2002) have argued that this is a clearly insufficient method because it is based on the fitting and isophotal analysis which can be affected by, e.g., nuclear clusters of star formation, or dust extinction. Regan \& Mulchaey (1999) and Martini \& Pogge (1999) have suggested to use the offset dust lanes - which are characteristic of large-scale bars, to detect nuclear bars. However, the dynamics of stellar secondary bars in these systems differs substantially from their large-scale counterparts (section 4.2) and no large-scale shocks will form under these conditions (Shlosman \& Heller 2002).

Laine et al. (2002) find that the distribution of normalized (to $D_{25}$ ) stellar nested bar sizes is bimodal. The minimal overlap between the distribution occurs at the (normalized to the galaxy size) bar length $\approx 0.06$. However, while primary bar sizes exhibit a roughly linear correlation with the parent galaxy sizes, the secondary bar sizes are independent from the sizes of their host galaxies. The importance of this result can be inferred from the fact that only in this case the normalized bar lengths will preserve the identity of both bar groups and there will be no further mixing between the primary and secondary bars in the normalized size space. This bimodal distribution can be understood within the framework of disk resonances, specifically the ILRs, which are located where the gravitational potential of the innermost galaxy switches effectively from 3D to 2D. This conclusion is further strengthened by the observed distribution of the sizes of nuclear rings which are dynamically associated with the ILRs. This could be the first observational evidence that the ILRs play the role of dynamical separators in disk galaxies.

While the gas contents of nuclear bars vary, in some cases, the cold gas can be dynamically important, as evident from the $2.6 \mathrm{~mm} \mathrm{CO}$ emission and NIR lines of $\mathrm{H}_{2}$ (e.g., Ishizuki et al. 1990; Devereux, Kenney \& Young 1992; Forbes et al. 1994; Mirabel et al. 1999; Kotilainen et al. 2000; Maiolino et al. 2000). Such nuclear gaseous bars have no large-scale counterparts in the local universe.

\subsection{Dynamics and dynamical decoupling of nested bars}

What makes the nested bars a truly unique dynamical system is their ability to be in coupled or decoupled states - the astrophysical counterpart of a coupled oscillator 
which is frequently invoked in studies of nonlinear behavior (e.g., Lichtenberg \& Lieberman 1995). The former state is characterized by identical pattern speeds of the primary and secondary bars, latter one - by substantially different pattern speeds. We note, that the decoupled states in galactic dynamics are not confined to nested bars only, but rather manifest the general importance of nonlinear physics in e.g., studies of spiral structure, interactions between the halos and the disks, and more. Although predicted theoretically and subsequently verified by numerical simulations, observations provide solid support for this idea. Friedli et al. (1996) have noted that the angle between the stellar nested bars is random, meaning that the observed bar systems are indeed found in the decoupled state. Furthermore, the first direct measurement of the pattern speeds in such bars has confirmed that these are different in NGC 2950 (Corsini et al. 2003).

The main question concerning dynamics of nested bars is what defines the pattern speed of the secondary bar after decoupling? The details of the actual decoupling process remain obscure. Finally, how efficient are these systems in channeling the gas inward and fueling the nuclear starbursts and AGN (sections 5, 6)? We, therefore, aim to verify the basic facts about nested bar dynamics in order to understand their potential ability to influence the overall dynamics in the central kpc.

Decoupling: pure stellar bars. Resonances play an important role in linear and especially in nonlinear dynamics. Typically, they increase the dissipation rate in the gas. But a less trivial and purely nonlinear effect is mode coupling. This applies to spiral and bar modes (e.g., Tagger et al. 1987). The basic idea is that nonlinear modes can exchange energies and angular momentum and hence can support pattern speeds which otherwise would decay exponentially. The question that interests us here is to what degree the nonlinear mode coupling can be responsible for the initial separation and the subsequent maintenance of different pattern speeds in nested bar systems.

Numerically, the decoupling of pure stellar bars is achieved through initial conditions only (e.g., Friedli \& Martinet 1993). With a sufficient number of particles, this state is long-lived. The pattern speed of the secondary bar puts its corotation radius at the position of the ILR of the primary bar. This pumps energy into the swing amplification cycle of the secondary bar. It is not clear, however, how the initial condition necessary for decoupling in this case can be achieved in the normal process of galactic evolution - as we shall see, the crucial ingredient, the gas, which is responsible for dissipation is missing in these calculations.

The real stellar nested bar systems are so abundant in the local universe that they must be long-lived. We discard the possibility that such systems can be built mostly from trajectories which transit between the two component bars. El-Zant \& Shlosman (2003) have shown that a typical nested bar system is made of regular orbits confined to each bar and trapped chaotic orbits in the vicinity of the regular regions. But what are the limits in the parameter space corresponding to such a stable system?

The middle horizontal panel in Fig. 3 shows a generic model of nested bars which appears to be stable, based on an analysis of the Liapunov exponents. Almost all orbits corresponding to the secondary bar are aligned with its major axis (middle vertical column) and appear regular (left column), most having axial ratios $p \geq 3$ (right). The top panel has a 4 times less massive secondary bar, and bar surface density ratio of only 2 , while most of the parameter space exhibits regular and trapped trajectories within the primary bar. The secondary bar did not produce orbits required to sustain it, and no orbits 


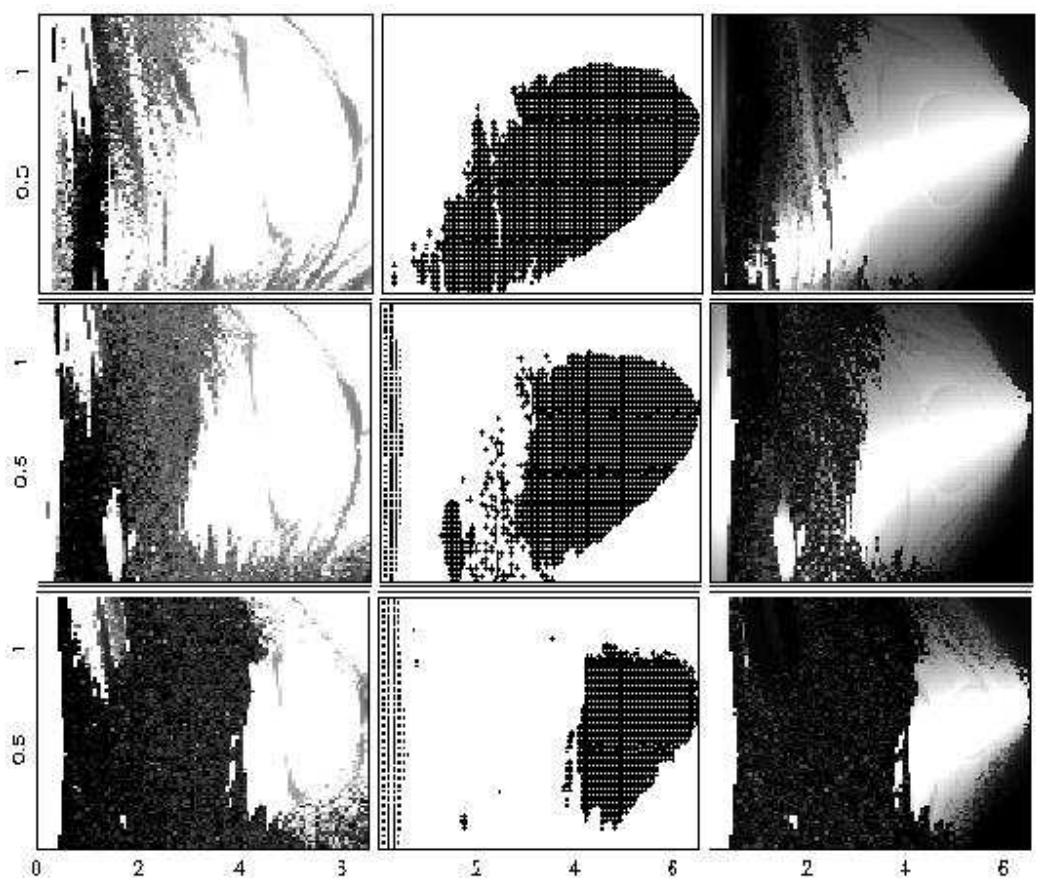

FIGURE 3. Double-bar galaxy models with secondary bar masses increasing from top to bottom by a factor of 4 each. The middle panels represent the generic model. The abscissa refers to radii (in kpc) and the ordinate to fractions of the local (circular) rotation velocity. Left column: Grayshades show the logarithms of Liapunov exponents (black-to-white corresponds to increased stability, from characteristic Liapunov time of $10^{2} \mathrm{Myr}$, i.e. chaotic orbits, to $10^{4} \mathrm{Myr}$, i.e. regular orbits). Middle column: Exhibits orbits supporting primary (crosses) and secondary (dots) bars, respectively. Right column: Maps the axial ratios, $p \equiv b / a$, of orbits (black-to-white grayshades correspond to increase from $p \leq 1$ to $p \geq 3$ ) (El-Zant \& Shlosman 2003).

aligned with it have been found for this model. Simultaneously, at the bottom panels, the chaotic region just outside the inner bar (bar-bar interface) expands, decreasing orbital support for the primary bar by no longer displaying significant alignment with it. This defines the critical mass fraction of the secondary bar, a few percent of that of the primary bar. Below this mass (and surface density) the inner bar is not sustainable, and above the outer bar support is dramatically weakened. On the basis of the left and middle panels of Fig. 3, we can safely rule out the top and the bottom panels, thus limiting the 'window of opportunity' for the stable existence of these systems.

The majority of the trajectories aligned with the primary bar and virtually all those aligned with the secondary bar are parented by families analogous to the single-periodic $x_{1}$ family in time-independent single-barred systems, some by higher order families. The symmetry of these orbits requires that one of the coordinates is maximal when the other is null (a variation of $10 \%$ on the exact values was introduced to allow for the effect of the perturbing bar). This has been verified by recording the $y$-coordinate of maximal $x$-excursion and vice versa. Between $1 / 3$ and $2 / 3$ orbits (from the top to bottom panels in Fig. 3) of trajectories have been found to be quasi-periodic regular ones, with exponential timescales of the order of a Hubble time or larger. They represent about 
$50 \%$ of trajectories in the generic model. Most $(50 \%-70 \%)$ of the regular trajectories are elongated in the direction of either bars, when viewed in the relevant frame.

However, a significant fraction of the trajectories supporting the bars includes trapped chaotic orbits. This is especially true in the case of the primary bar, where such trapped orbits constitute about $16 \%$ of the supporting trajectories in the generic model. Their fraction peaks at $20 \%$ when the secondary bar mass is halved, while they are quickly replaced by "strongly chaotic" orbits when the mass is doubled. Although the trapped trajectories have a non-zero Liapunov exponent, many of them mimic bar-supporting orbits for a Hubble time or so. In general, trapped chaotic trajectories may wander intermittently between regular and chaotic phases with a distribution described by nonstandard statistics (Zaslavsky 2002). If the initial conditions are such that a significant number of these trajectories are in a trapped phase, they may be of crucial importance to building such systems as double-barred galaxies.

Decoupling: non-self-gravitating gaseous bars. Gaseous bars have attracted much less attention than their stellar counterparts, primary and secondary. The simplest explanation is that gas rarely dominates the dynamics of stellar disks beyond the local Jeans instability. In addition, numerical modeling of gaseous bars requires a large dynamic range, as shown below - the gaseous bars quickly self-destruct when they become selfgravitating. However, it is exactly this fast process which is of great interest in studies of fueling the central activity whether of starburst of AGN type.

We start with a simplest case by adding a low surface density (i.e., non-selfgravitating) gas to a galactic disk with a single large-scale bar and a double ILR. The gas response leads to the formation of nuclear rings between the ILRs (or inside the ILR, if only one is present). One should keep in mind also that the torques change their sign at the IILR. The periodic orbits between the ILRs (or inside the single ILR), so-called $x_{2}$ orbits $^{1}$ (Contopoulos \& Papayannopoulos 1980), are oriented perpendicularly to the primary bar and serve as attractors to gas motions in their vicinity. We note that the gas distribution in the region is never symmetric with respect to the primary bar axis, with the nuclear ring oriented in the first and third quadrants - this leads to a constant drain of angular momentum from the gas by the primary bar and to a progressively more elongated ring.

Hence it is not surprizing that a large fraction of cold (molecular) gas piles up in ILR(s) region on the $x_{2}$ orbits, independently of the presence or absence of selfgravitational effects in the gas. The simplest type of decoupling between the gas and the primary bar is not driven by the gas self-gravity (Heller, Shlosman \& Englmaier 2001). Instead, the gaseous ring drifts deeper into the potential well because of internal dissipation. As the gas crosses the IILR, it loses the attractor ${ }^{2}$ (i.e., the $x_{2}$ orbits), which leads to a retrograde precession (in the primary bar frame) because of the mutual barring orientations. One possibility is that the gaseous ring/bar remains trapped by the primary bar potential valley - this results in secondary bar libration about the major

\footnotetext{
${ }^{1}$ Here we mean fully nonlinear orbits, not in the epicyclic approximation, as they have been introduced originally

${ }^{2}$ Stricktly speaking, the gas does not follow the periodic orbits, $x_{1}$ or $x_{2}$, rather those serve as attractors. Viscous torques prevent the gas from being completely aligned with the periodic orbits
} 
axis of the primary. More spectacular is a complete decoupling of the secondary and its substantial slowdown - the gaseous bar tumbles in the retrograde direction (in the primary frame) until it is captured again by the primary potential. This effect is caused by the gravitational torques from the primary bar and the ability of the secondary to adjust its shape (ellipticity) depending on the mutual orientation of the bars. This type of behavior can lead to a mild inflow across the IILR and associated bursts of star formation.

Decoupling: self-gravitating gaseous bars. Modeling self-gravitating gaseous bars in nested systems is more difficult. Decoupling was not obtained for this case in numerical simulations by Wada \& Habe (1992) and Combes (1994). Understandably, gas exhibits the strongest response to the galactic bar potential which triggers its inflow toward the central regions. While the gas accounts only for a few percent of the mass within the luminous part of a galaxy, its contribution is expected to rise by a factor of $\sim 10$ within the central kpc. Because of this and because the random motions within this gas are substantially lower than within the stellar disk, the gas can be dynamically important within the central $\mathrm{kpc}$ - a property which is amplified even further due to the gas clumpiness. One expects the gas to settle on the $x_{2}$ orbits within ILR(s) region in a nuclear ring or disk, whether one or two ILRs exist - depending on how cuspy the mass distribution is within the central kpc.

Settling the gas on $x_{2}$ orbits, therefore, appears to be a general outcome of bar-driven gas inflow towards the center. Even if no ILRs exist there initially, the disk rotation curve which is modified by the gas gravity will form at least one ILR. The question is how the addition of gravity will change the gas dynamics compared with the case discussed above. Fig. 4 shows an initial evolution which proceeds along the lines of the case for non-self-gravitating gas. Even the initial retrograde decoupling appears similarly because the gas mass fraction is $\lesssim 2 \%$ within corotation of the primary bar in this model. However, this is where the evolution of both types of models diverges. A short time after decoupling the gaseous (secondary) bar collapses dramatically by a factor of $\sim 7$ and accelerates in the prograde direction by about the same factor (Fig. 5). Its pattern speed stabilizes thereafter and the system persists in this decoupled state.

Analysis of this model provides some insight into the decoupling process and the special role of gas in it. First, the retrograde decoupling is of no principal importance here - an increase of $\sim 20 \%$ of gas mass results in a purely prograde decoupling without the retrograde phase. Second, the final steady state in the model follows from a finite dynamic range in the numerical model - the limiting gravitational softening of $150 \mathrm{pc}$. This is the end-size of the secondary bar after the catastrophic inflow and this apparently determines its pattern speed.

What is more important is that the secondary bar ellipticity (i.e., its strength) increases beyond 0.8 - its axial ratio becomes $b / a<1: 5$. This exceptional increase in the bar strength also serves as the main reason for its demise. In an early paper by Contopoulos (1981), the increase in the non-axisymmetric part of the bar potential has been related to the onset of stochasticity in the bar. More generally, stronger bars destabilize the major families of orbits supporting the bar which become progressively chaotic. Unstable regions associated with resonances widen and start to overlap. The previously local chaos becomes global and the system dissolves. The dissolution of strong collisionless bars, with axis ratio $1: 5$ and below, has been modeled (e.g., Teuben \& Sanders 1985). 


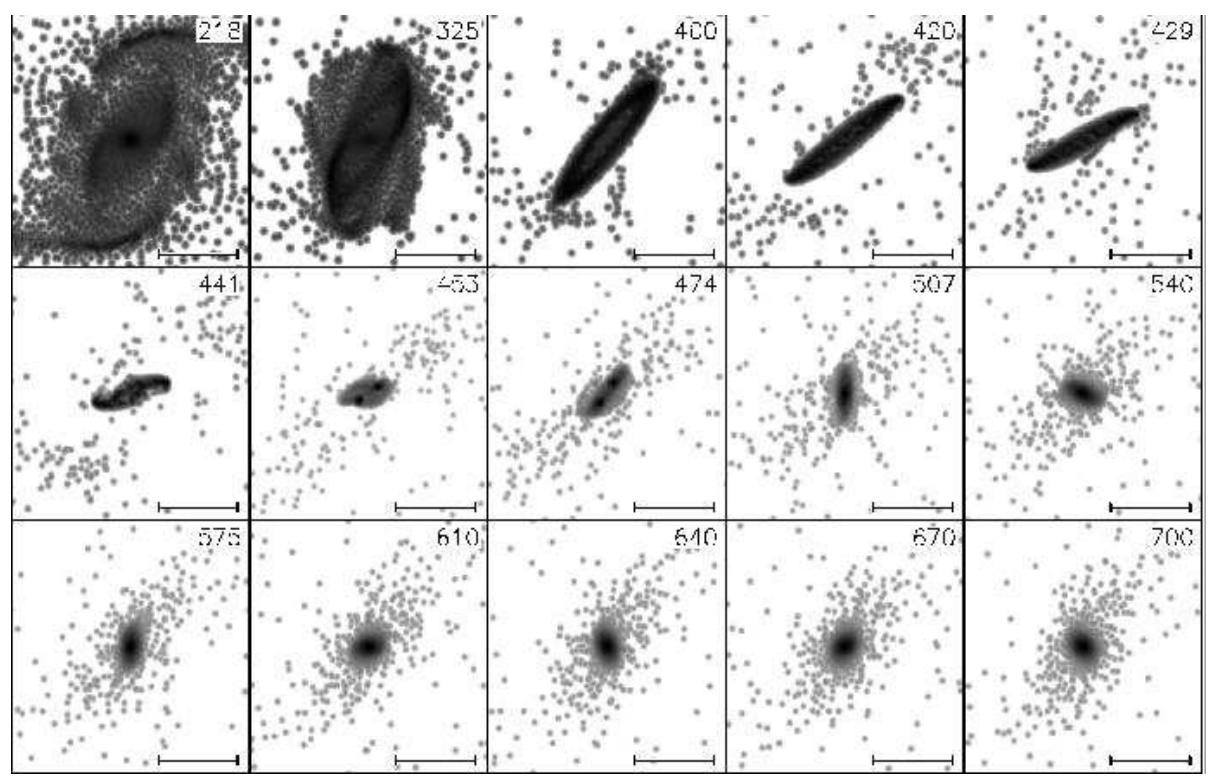

FIGURE 4. Decoupling of self-gravitating nested bars: $6 \mathrm{kpc}$ stellar (primary) bar (not shown) and $1 \mathrm{kpc}$ gaseous (secondary) bar. Each frame shows central $3 \mathrm{kpc} \times 3 \mathrm{kpc}$ part of a barred galaxy in the frame of the primary bar (horizontal). Rotation is anti-clockwise. Time in Myrs is shown in the right upper corners and the horizontal bar is $1 \mathrm{kpc}$ in length. Gray scale represents the gas density (Englmaier \& Shlosman 2004).
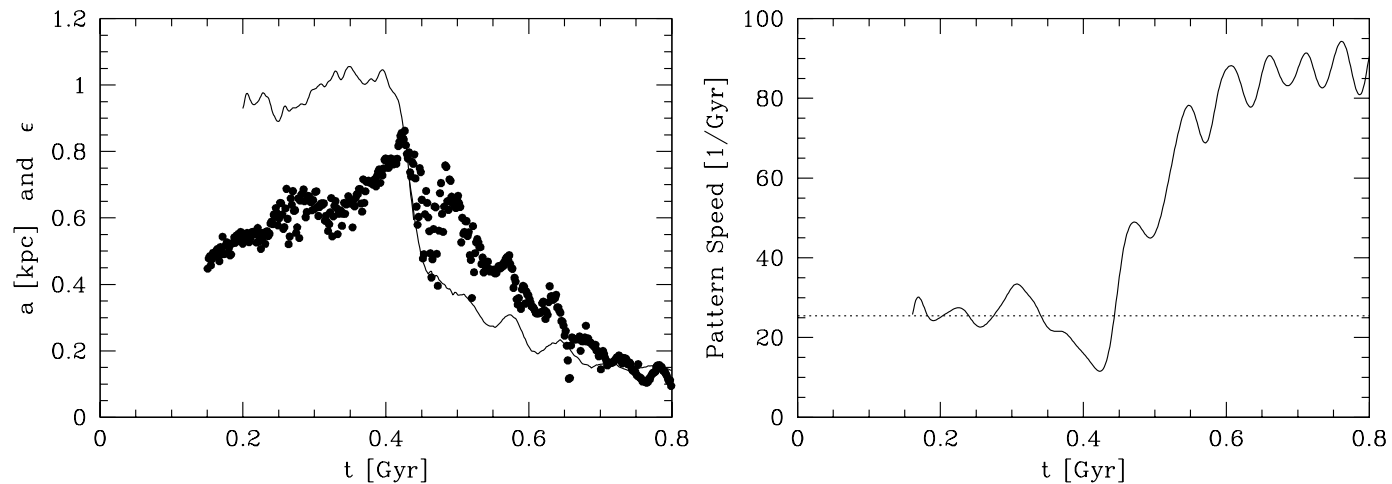

FIGURE 5. Left: Evolution of ellipticity, $\varepsilon=1-b / a$ (dotted line), and semimajor axis, $a$ (solid line) of the secondary bar. Right: Pattern speeds of primary $\left(\Omega_{\mathrm{p}}\right.$, dots) and secondary $\left(\Omega_{\mathrm{s}}\right.$, solid line) bars in the inertial frame (Englmaier \& Shlosman 2004).

The situation is expected to be even more dramatic in self-gravitating gaseous bars because of a two-fold reason. First, gaseous bars can reach an even smaller axial ratio due to the dissipation. Second, the gas cannot reside on intersecting orbits - hence the increase in the fraction of chaotic orbits should have catastrophic consequences for gaseous bars. Fig. 5 confirms this overall behavior.

The main difference between gaseous and stellar secondary bars is this ability of the former to contract dramatically due to internal shocks. A number of triggers which are capable to excite these shocks can be listed, however it is plausible that the increased 
bar strength and the associated growth of the fraction of chaotic orbits is sufficient to cause this runaway. But how does decoupling proceed? This bar strength is a direct consequence of gas self-gravity. The test model with non-self-gravitating gas shows a diverging evolution - it never decouples in the prograde direction.

Based on a large number of models, we find that the decoupling process approximately preserves the product of secondary bar pattern speed, $\Omega_{\mathrm{s}}$, and the bar size, $a$, i.e., $\Omega_{\mathrm{s}} a \sim$ const. (Englmaier, Shlosman \& Heller 2004). This means that the decoupling gas is losing its angular momentum, $\Omega_{\mathrm{s}} a^{2}$, to the primary bar. But it also means that $\Omega_{\mathrm{s}}$ is increasing sharply during this phase. One can make an analogy between this behavior of the gaseous bars and evolution of single stellar bars. The latter are known to extend to their corotation (e.g., Athanassoula 1992) which typically lies in the flat part of the galactic rotation curve, and so $\Omega(r) \sim r^{-1}$. Pure stellar bars embedded in live halos are transferring their angular momentum to these halos and as a result slow down their pattern speed, $\Omega_{\mathrm{p}}$. Their growth provides a partial 'compensation' for the loss of angular momentum, hence $\Omega_{\mathrm{p}} r_{\mathrm{p}} \sim$ const., where $r_{\mathrm{p}}$ stands for the stellar bar size. A similar analysis performed in the epicyclic approximation for the secondary bars leads to the same relation between the pattern speed and the bar size, if the bar extends to the position of the IILR - which is what is observed in our numerical simulations. We note this is only possible if the gas contributes a non-negligible fraction of the mass within its radius $(\sim 10 \%-16 \%$ in our models) because only in this case the contracting gas bar pulls the IILR inward.

Two important questions follow from the above discussion: what determines the final $\Omega_{\mathrm{s}}$, and to what degree can the mode coupling influence the whole process. The speedup of the secondary gaseous bar clearly depends on its ability to contract and, therefore, to dissipate the internal (circulation) angular momentum. In the modeling, this is limited by the dynamic range of the numerical scheme and to a certain degree by the equation of state for the gas. We have used limiting gravitational softening of $150 \mathrm{pc}$, which determines the final semimajor axis of the bar. Of course, further decrease in the softening value requires knowledge of the equation of state on smaller spatial scales or an additional energy source, e.g., associated with supernovae, to prevent the development of a Jeans instability. An alternative treatment is to introduce a multi-phase ISM (e.g., Wada $\&$ Norman 2002). Hence, in principle, we can argue that the contraction of gaseous bars in nested systems can proceed to substantially smaller spatial scales than discussed here, if the issue of limiting softening is overcome. In principle, unless additional physical phenomena become important, this type of contraction will lead to exceedingly smaller and denser gas bars with substantially higher pattern speeds and it is not clear at all if an upper limit exists for this evolutionary stage. This behavior of course has direct consequences for fueling of nuclear starbursts and of AGN (section 5).

While we do not find that the mode coupling plays a role in the runaway, it can be important in locking $\Omega_{\mathrm{s}}$ with $\Omega_{\mathrm{p}}$, if the bar contraction is stopped for some reason. On the other hand, the avalanche contraction of the gaseous bar can be related to the selforganized criticality - a state which is an attractor for gas dynamics within the bar. ${ }^{3}$

\footnotetext{
${ }^{3}$ In this framework, an analogy can be made between the bar strength-driven chaos and the sandpile whose slope is increased until the sand slides off (e.g., Lichtenberg \& Lieberman 1995).
} 


\section{GAS FLOWS IN NESTED BARS AND THE FUELING OF AGN}

We can distingush between two types of nested bars based on their support of radial gas flows: those secondary bars are of mixed or purely gaseous types. The gas dynamics differs profoundly between these two cases. The role of these nested systems in fueling the central activity, thermal and nonthermal, is now being investigated by a number of research groups, and so is their effect on the overall galaxy evolution.

If the background potential is dominated by stars, the gas inflow can be driven either by a time-dependent potential or by large-scale shocks delineated by the dust lanes, e.g., as in primary stellar bars. However, these large-scale shocks are not detected in the secondary bars and one can understand why. If the secondary bars extend to their corotation - a condition necessary for the formation of large-scale shocks - they will feel a strongly time-dependent potential in the outer bar-bar interface. This region will not be able to retain the gas injected by the primary bar and the gas would 'fall through' (Heller, Shlosman \& Englmaier 2001). If, on the other hand, the bars do not extend to their corotation, the shocks will not form either. This of course does not preclude the gas flows across the bar-bar interface, it makes it largely chaotic but also correlated with the bars' mutual orientation. The gas then settles in the inner part of the secondary bar and its future evolution is more similar to that of gas-dominated bars (see below).

The above statement applies of course to fully decoupled bars. Before the decoupling, when the bars corotate, the secondary bars can extend to their corotation. But the offset shocks in the gas which settles on the $x_{2}$ orbits will be absent as well.

Gas inflows in the star-dominated secondary bars can be recurrent, depending on the external fuel supply. This means that the system can exist in the decoupled phase for long periods of time without any gas transfer to the center. On the other hand, in the periods of active inflow, it is unclear whether there will be substantial star formation, which can be inhibited by local shear, as in primary bars. The corollary is that stellar nested bars most probably will contain an old stellar population, which dominates even in the secondary bars, in agreement with observations by Friedli et al. (1996) of a few stellar nested bars galaxies. This is an important point and shows that in secondary stellar bars, as in large-scale primary bars, the age of the stellar population has nothing to do with its ability to channel gas to smaller spatial scales.

If the background potential of the secondary bar is dominated by the gas, the inflow is triggered by the growing chaos in this bar, as we have discussed above. The avalanchetype inflow proceeds on the crossing timescale and the inflow rates increase with decreasing radius and can be of the order of quasar-type fueling rates (Fig. 6). Clearly, these inflows are closely associated with dissipation and compression in the gas. As such, they can be accompanied by bursts of star formation.

With the current understanding that SBHs in galactic centers are ubiquitous, at least in the nearby universe, issues related to the availability of fuel and its delivery mechanism(s) take central stage. The delivery mechanism is tied to the angular momentum problem (e.g., Shlosman et al. 1989; Jogee 2004) and a number of solutions have been proposed to resolve it. While the idea that nonlocal viscosity in the form of gravitational torques is responsible for fueling the central acitivity, either starburst and/or AGN-type, has acquired substantial theoretical and observational evidence in its favor, open issues remain. In particular, within the nested bar framework, the weak point appears to be 


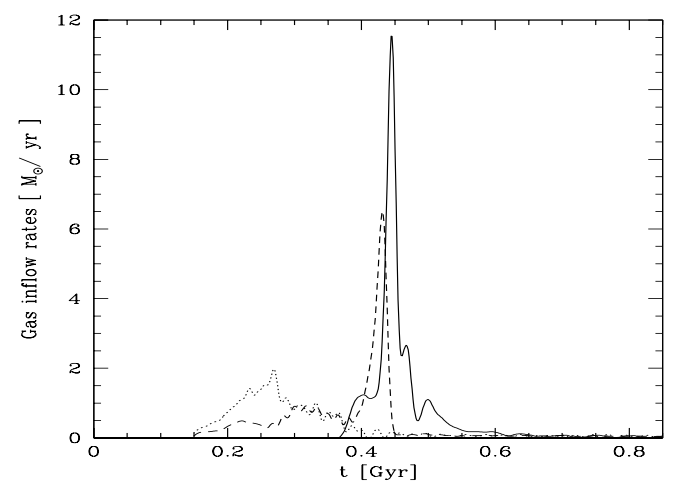

FIGURE 6. Mass inflow rates in self-gravitating gaseous secondary bars in decoupled nested systems. Dotted line corresponds to the inflow across $1 \mathrm{kpc}$, dashed line - across the inner $600 \mathrm{pc}$, and solid line — across the central 175 pc (Englmaier \& Shlosman 2004).

the lack of statistics of gaseous nuclear bars. The evolution shown here favors very short timescales of $\sim 10^{7} \mathrm{yrs}$, which makes detection of such systems difficult. On the other, single bars, spontaneous or tidally-induced by mergers and interactions, are capable of transferring the gas by only a decade in radius. This is a clear limitation on the efficiency of large-scale stellar bars in fueling the central activity, and the need for additional mechanism(s) operating within the central $\mathrm{kpc}$ was understood already more than a decade ago (Shlosman et al. 1989, 1990). It is surprizing, therefore, that Wada (2004) claims that no need exists for triggers of nuclear activity, by e.g., counting such alternatives as a radiative avalanche (which operates on a timescale by far exceeding the Hubble time). We find that while the uniqueness of the mechanism discussed here is open, solid observational and theoretical arguments point out that the importance of nested bar systems is not only limited to the fueling issue but is essential for the overall evolution of galactic disks as well.

\section{COMPOSITE SEYFERT NUCLEI: A BY-PRODUCT?}

One of the focal questions in AGN fueling is to what extent AGN and nuclear starburst phenomena overlap and whether they are in any causal relationship. A fraction of Seyferts, commonly known as composite Seyferts, exhibit thermal emission from a starburst in addition to a nonthermal nuclear component (e.g., Miley et al. 1985), which is especially pronounced in Seyfert 2s (Edelson, Malkan \& Rieke 1987; Heckman et al. 1989). Taken in tandem with the measured excess of CO emission, this may hint in the direction of an evolutionary sequence: nuclear starburst $\rightarrow$ Seyfert $2 \rightarrow$ Seyfert 1 and hence provide a "missing" link between starbursts and AGN, as first suggested by Heckman et al. (1989), and recently advanced by Storchi-Bergmann et al. (2001). The key question is somewhat broader in significance: even if there is a basis for this temporal sequence, can we assume that the nuclear starburst is the cause of AGN fueling and not merely a by-product of radial gas inflow?

The recent detection of spectroscopic signatures of hot massive stars in a number 
of Seyfert 2 nuclei has provided additional support for the ongoing debate on a AGNstarburst connection (e.g., Knapen et al. 2001). These composite Seyfert 2 nuclei exhibit stellar wind lines, W-R features and high-order Balmer absorption lines superposed on their "featureless" AGN continua.

The smallest of these starbursts, when spatially resolved, have effective radii between 55 pc and 200 pc (González-Delgado et al. 1998) and their morphology closely resembles that of nuclear starbursting rings. The phenomenon of nuclear rings in disk galaxies is fairly well studied and understood (e.g., Athanassoula 1992; Knapen et al. 1995; Heller \& Shlosman 1996; Knapen 2005). If indeed these resolved nuclear starbursts are representative of all the composite Sy nuclei, their origin is unrelated to the central AGN. On the other hand, it provides the clearest indication of a recent gas inflow on the spatial scales these rings are found. So the relevant physics to understand this phenomenon will be the galactic (gas) dynamics and not the AGN physics. In this case, the starburst is clearly a by-product of gas redistribution in the galaxy.

The nuclear rings can be associated with OILR, IILR and NLR (i.e., nuclear LR) (e.g., Shlosman 1999 for review). The OILR ring is never positioned at the resonance but rather halfway between the OILR and IILR, while the innermost ring sits exactly at the IILR. Numerical simulations show that if there is more than one nuclear ring, they interact and merge and the resulting ring appears at the IILR due to the change of sign in gravitational torques there. This resonance is usually found close to the center and, therefore, the observed starburst in composite Sy nuclei can be related to the IILR.

\section{CONCLUSIONS}

While it is clear that the issues reviewed here are not resolved completely, substantial progress has been made on all spatial scales, from $\sim 1-30 \mathrm{pc}$ - the size of the molecular tori in AGN, to $\gtrsim 10 \mathrm{kpc}$ - that of the DM halos and well beyond. A number of corollaries gradually become obvious: the formation and evolution of galaxies appears to be a much more correlated process compared to what had been envisioned before, despite the enormous dynamic range and dissipation involved. This coupling between different parts of SBH-disk-halo systems emerges as strongly nonlinear, largely due to the nonlocal gravitational viscosity involved and possibly due to mode coupling. On the other hand, this nonlinearity results in a non-negligible contribution of chaotic motions in building the structure of the luminous part of disk galaxies and to a new phenomenon of dynamical decoupling of different parts in these galaxies.

Acknowledgements. I am indebted to my colleagues for numerous and lengthy discussions on different issues concerning this work, especially to Mitch Begelman, Ingo Berentzen, Amr El-Zant, Peter Englmaier, Clayton Heller, Shardha Jogee, Johan Knapen and Barbara Pichardo. I acknowledge partial support by NASA/ATP/LTSA grants NAG5-10823, 5-13063, by NSF AST-0206251, and by HST AR-10284. 


\section{REFERENCES}

1. Abraham, R.G., Merrifield, M.R., Ellis, R.S., Tanvir, N.R., Brinchmann, J. 1999, MNRAS, 308, 569

2. Antonucci, R., 2002, Astrophysical Spectropolarimetry, ed. J. Trujillo-Bueno et al. (CUP), 151

3. Arav, N., Shlosman, I., Weymann, R.J., eds. 1997, Mass Ejection from AGN (ASP Conf. Ser.)

4. Athanassoula, E. 1984, Phys. Reports, 114, 319

5. Athanassoula, E. 1992, MNRAS, 259, 345

6. Binney, J., Tremaine, S. 1987, Galactic Dynamics. Princeton University Press

7. Blandford, R.D., Payne, D.G. 1982, MNRAS, 199, 883

8. Bock, J.J., et al. 2000, AJ, 120, 2904

9. Borriello, A., Salucci, P. 2001, MNRAS, 323, 285

10. Bosma, A. 2002, Disks of Galaxies: Kinematics, Dynamics \& Interactions, (eds.) E.Athanassoula et al. (ASP Conf. Ser. 275), 23

11. Bottorff M.C., Korista, K.T., Shlosman, I., Blandford, R.D. 1997, ApJ, 479, 200

12. Bottorff, M.C., Korista, K.T., Shlosman, I. 2000, ApJ, 537, 134

13. Burkert, A. 1995, ApJ, 447, L25

14. Cole, S., Lacey, C. 1996, MNRAS, 281, 716

15. Combes, F., Gerin, A. 1985, A\&A, 150, 327

16. Combes, F. 1994, Mass-Transfer Induced Activity in Galaxies, ed. I.Shlosman (CUP), 170

17. Contopoulos, G., Papayannopoulos, T. 1980, A\&A, 92, 33

18. Contopoulos, G. 1981, A\&A 102, 265

19. Contopoulos, J. 1995, ApJ, 446, 67

20. Corsini, E.M., Debattista, V.P., Aguerri, J.A.L. 2003, ApJ, 599, L29

21. de Block, W.J.G., Bosma, A. 2002, A\&A, 385, 816

22. Devereux, N.A., Kenney, J.D., Young, J.S. 1992, AJ, 103, 784

23. Dubinski, J., Carlberg, R.G. 1991, ApJ, 378, 496

24. Dubinski, J. 1994, ApJ, 431, 617

25. Edelson, R.A., Malkan, M.A., Rieke, G.H. 1987, ApJ, 321, 233

26. El-Zant, A., Shlosman, I., Hoffman, Y. 2001, ApJ, 560, 636

27. El-Zant, A., Shlosman, I. 2003, ApJ, 595, L41

28. Elitzur, M., Nenkova, M., Ivezić, Z. 2004, The Neutral ISM in Starburst Galaxies, eds. S. Aalto, S. Hüttemeister \& A. Pedlar, ASP Conf. Proc. 320, in press (astro-ph/0309040)

29. Emmering, R.T., Blandford, R.D., Shlosman, I. 1992, ApJ, 385, 460

30. Englmaier, P., Shlosman, I. 2000, ApJ, 528, 677

31. Englmaier, P., Shlosman, I. 2004, ApJ Letters, 617, in press (astro-ph/0410283)

32. Englmaier, P., Shlosman, I., Heller, C.H. 2004, ApJ, sibmitted

33. Erwin, P., Sparke, L.S. 2002, AJ, 124, 65

34. Ferrarese, L., Ford, H. 2004, astro-ph/0411247

35. Flores, R.A., Primack, J.R. 1994, ApJ, 427, L1

36. Forbes, D.A., Kotilainen, J.K., Moorwood, A.F.M. 1994, ApJ, 433, L13

37. Friedli, D., Martinet, L. 1993, A\&A, 277, 27

38. Friedli, D., Wozniak, H., Rieke, M., Martinet, L., Bratschi, P. 1996, A\&AS, 118, 461

39. Fukushige, T., Makino, J. 1997, ApJ, 477, L9

40. Gonzalez-Delgado, R.M., et al. 1998, ApJ, 505, 174

41. Hawley, J.F., Gammie, C.F., Balbus, S.A. 1996, ApJ, 464, 690

42. Hawley, J.F., Balbus, S.A., Stone, J.M. 2001, ApJ, 554, L49

43. Heckman, T.M., Beitz, L., Wilson, A.S., Armus, L., Miley, G.K. 1989, ApJ, 342, 735

44. Heller, C.H., Shlosman, I. 1994, ApJ, 424, 84

45. Heller, C.H., Shlosman, I. 1996, ApJ, 471, 143

46. Heller, M.C., Shlosman, I., Englmaier, P. 2001, ApJ, 553, 661

47. Hubble, E.P. 1936, The Realm of the Nebulae, New Haven: Yale Univ. Press

48. Hüttemeister, S. 2002, Disks of Galaxies: Kinematics, Dynamics \& Interactions, (eds.) E.Athanassoula et al. (ASP Conf. Ser. 275), 243

49. Ishizuki, S., Kawabe, R., Ishiguro, M., Okumura, S.K., Morita, K.-I. 1990, Nature, 344, 224

50. Jogee, S. 2004, AGN Physics on All Scales, Eds. D. Alloin, R. Johnson \& P. Lira (Springer: Berlin), Ch. 6, in press (astro-ph/0408383) 
51. Jogee, S., Scoville, N.Z., Kenney, J. 2004a, ApJ, in press (astro-ph/0402341)

52. Jogee, S., Barazza, F.D., Rix, H.-W., Shlosman, I., et al. 2004b, ApJ, 615, L105

53. Kartje, J.F., Königl, A., Elitzur, M. 1999, ApJ, 513, 180

54. Klypin, A., Kravtsov, A., Colin, P. 2000, Cosmic Flows Workshop, ASP Conf. Ser. 201, 344

55. Knapen, J.H. 2005, A\&A, in press (astro-ph/0409031)

56. Knapen, J.H., Beckman, J.E., Heller, C.H., Shlosman, I., de Jong, R.S. 1995, ApJ, 454, 623

57. Knapen, J.H., Beckman, J.E., Shlosman, I., Mahoney, T.J. (eds.) 2001, The Central Kpc of Starbursts

\& AGN: the La Palma Connection, ASP Conf. Ser., Vol. 249

58. Königl, A., Kartje, J.F. 1994, ApJ, 434, 446

59. Kotilainen, J.K., Reunanen, J., Laine, S.. Ryder, S.D. 2000, A\&A, 353, 834

60. Kravtsov, A.V., Klypin, A.A., Bullock, J.S., Primack, J.R. 1998, ApJ, 502, 48

81. Krolik, J.H., Begelman, M.C. 1988, ApJ, 329, 702

62. Laine, S., Knapen, J.H., Perez-Ramirez, D., Doyon, R., Nadeau, D. 1999, MNRAS, 302, L33

63. Laine, S., Shlosman, I., Knapen, J.H., Peletier, R.F. 2002, ApJ, 567, 97

64. Lake, G., Norman, C.A. 1983, ApJ, 270, 51

65. Lichtenberg A.J., Lieberman M.A., 1995, Regular and Chaotic Dynamics. Springer, New York

66. Lokas, E.L., Hoffman, Y. 2000, ApJ, 542, L139

67. Maiolino, R., et al. 2000, ApJ, 531, 219

68. Martini, P., Pogge, R.W. 1999, AJ, 118, 2646

69. Mathur, S., Elvis, M., Singh, K.P. 1995, ApJ, 455, 9

70. Mathur, S., et al. 2000, ApJ, 533, L79.

71. McGaugh, S.S., Rubin, V.C., de Block, W.J.G. 2001, AJ, 122, 2381

72. Miley, G.K., Neugebauer, G., Soifer, B.T. 1985, ApJ, 293, L11

73. Mirabel, I.F., et al. 1999, A\&A, 341, 667

74. Moore, B., Quinn, T., Governato, F., Stadel, J., Lake, G. 1999, MNRAS, 310, 1147

75. Navarro, J.F., Frenk, C.S., White, S.D.M. 1996, ApJ, 462, 563 (NFW)

76. Nenkova, M., Ivezic, Z., Elitzur, M. 2002, ApJ, 570, L9

77. Pichardo, B., Shlosman, I. 2005, in preparation

78. Radomski, J.T. et al. 2003, ApJ, 587, 117

79. Regan, M.W., Mulchaey, J.S. 1999, AJ, 117, 2676

80. Rix, H.-W., et al. 2004, ApJS, 152, 163

81. Sakamoto, K., Okumura, S.K., Ishizuki, S., Scoville, N.Z. 1999, ApJ, 525, 691

82. Sand, D.J., Treu, T., Ellis, R.S. 2002, ApJ, 574, 129

83. Schwarz, M.P. 1984, MNRAS, 209, 93

84. Shields, J.C., Hamann, F. 1997, ApJ, 481, 752

85. Shlosman, I., Frank, J., Begelman, M.C. 1989, Nature 338, 45

86. Shlosman, I., Begelman, M.C., Frank, J. 1990, Nature 345, 679

87. Shlosman, I., Noguchi, M. 1993, ApJ, 414, 474

88. Shlosman, I. 1999, Evolution of Galaxies on Cosmological Timescales,(eds.) J.E. Beckman \& T.J.

Mahoney (ASP Conf. Series, Vol. 187), 100

89. Shlosman, I. 2001, The Central Kpc of Starbursts \& AGN, J.H. Knapen et al. (ASP Conf. Ser.), 55

90. Shlosman, I., Heller, C.H. 2002, ApJ, 565, 921

91. Simkin, S.M., Su, H.J., Schwarz, M.P. 1980, ApJ, 237, 404

92. Storchi-Bergmann, T., et al. 2001, ApJ, 559, 147

93. Tagger, M., Sygnet, J.F., Athanassoula, E., Pellat, R. 1987, ApJ, 318, L43

94. Teuben, P.J., Sanders, R.H. 1985, MNRAS, 212, 257

95. Treister, E., et al. 2004, ApJ, in press (astro-ph 0408099)

96. Wada, K., Habe, A. 1992, MNRAS, 258, 82

97. Wada, K., Norman, C.A. 2002, ApJ, 566, L21

98. Wada, K. 2004, Coevolution of Black Holes and Galaxies, (ed.) L.C. Ho (CUP), 187

99. Wada, K., Tomisaka, K. 2005, ApJ, 20 Jan., 2005, in press (astro-ph/0410254)

100. Warren, M.S., Quinn, P.J., Salmon, J.H., Zurek, W.H. 1992, ApJ, 399, 405

101. Weigelt, G. et al. 2004, A\&A, in press

102. Zaslavsky, G.M. 2002, Phys. Reports, 371, 461 